\title{
CYCLIC APPROXIMATION OF IRRATIONAL ROTATIONS
}

\author{
A. IWANIK
}

(Communicated by Andrew Bruckner)

\begin{abstract}
We prove that an irrational number $\alpha$ admits a rational approximation $|\alpha-p / q|=o(f(q))$ iff the irrational rotation $T x=\{x+\alpha\}$ admits cyclic approximation with speed $o(f(n))$. As an application to earlier results we obtain that a generic Anzai skew product over every irrational rotation is rank-1 and for a.e. $\alpha$ most skew products admit cyclic approximation with speed $o\left(1 / n^{2} \log n\right)$.
\end{abstract}

\section{INTRODUCTION}

Let $(X, \mu)$ be a Lebesgue space. If $\xi_{n}$ is a sequence of (finite measurable) partitions then we write $\xi_{n} \rightarrow \varepsilon$ if for any measurable set $A$ there exists a union $A_{n}$ of cells of $\xi_{n}$ such that $\mu\left(A \triangle A_{n}\right) \rightarrow 0$.

Let $0<f(n) \rightarrow 0$. According to Katok and Stepin [5] we say that an invertible measure-preserving transformation $T$ of $X$ admits a cyclic approximation with speed $o(f(n))$ if there exists a sequence of partitions $\xi_{n} \rightarrow \varepsilon$ and a transformation $T_{n}$ cyclically permuting the cells of $\xi_{n}$ such that

$$
\sum_{j=0}^{h_{n}-1} \mu\left(T C_{j} \triangle T_{n} C_{j}\right)=o\left(f\left(h_{n}\right)\right),
$$

where $\xi_{n}=\left\{C_{0}, \ldots, C_{h_{n}-1}\right\}, C_{j}=T_{n}^{j} C_{0}$.

It is well known that cyclic approximation with speed $o(1 / n)$, called good cyclic approximation, implies rigidity, singular simple spectrum $($ see $[5,4])$, and rank 1 of $T$.

As was pointed out in $[5, \S 1,1)$ and 2)] for an a.e. irrational number $\alpha$ the irrational rotation $T x=\{x+\alpha\}$ (where $\{x\}$ denotes the fractional part of $x$ ) of the unit interval $[0,1)$ admits a good cyclic approximation. In fact, it is easy to see that if $\left|\alpha-p_{n} / q_{n}\right|=o\left(1 / q_{n}^{2}\right)$ then $T_{n} x=\left\{x+p_{n} / q_{n}\right\}$ and $C_{0}=\left[0,1 / q_{n}\right)$ will do.

It is our aim to prove that the speed of cyclic approximation of the rotation $T$ is essentially as good as the speed of rational approximation of $\alpha$ (see Theorem 1). In particular, every irrational rotation admits a good cyclic approximation (this was observed earlier by del Junco, see [2]), while for a.e. $\alpha$ an admissible

Received by the editors August 7, 1992.

1991 Mathematics Subject Classification. Primary 28D05. 
speed is $o\left(1 / n^{2} \log n\right)$ (see Corollary). Our argument is similar to that in del Junco [1]; now, however, we have to ensure an error term of order $o\left(f\left(h_{n}\right)\right)$. Instead of using cyclic partitions for rational rotations we exploit properties of continued fractions and use two disjoint $T$-stacks covering the interval.

\section{CYCLIC APPROXIMATION}

Let $\alpha$ be an irrational number. We denote by $p_{n} / q_{n}$ its continued fraction approximation. It is well known that $\left|\alpha-p_{n} / q_{n}\right|<1 / q_{n}^{2}$. Moreover,

$$
q_{n+1}\left\|q_{n} \alpha\right\|+q_{n}\left\|q_{n+1} \alpha\right\|=1,
$$

where $\|x\|$ denotes the distance from $x$ to the nearest integer. This formula reflects splitting the interval into two Rokhlin towers $\zeta_{n}$ and $\zeta_{n}^{\prime}$ for the irrational rotation $T x=\{x+\alpha\}$. More precisely, if $n$ is even then $\zeta_{n}$, the large tower, consists of the sets $J_{j}=\left[\{j \alpha\},\left\{\left(q_{n}+j\right) \alpha\right\}\right), j=0, \ldots, q_{n+1}-1$, while $\zeta_{n}^{\prime}$, the small one, consists of $J_{0}^{\prime}=\left[\left\{q_{n+1} \alpha\right\}, 1\right)$ and $J_{j}^{\prime}=\left[\left\{\left(q_{n+1}+j\right) \alpha\right\},\{j \alpha\}\right)$, $j=1, \ldots, q_{n}-1$.

Theorem 1. Let $0<f(n) \rightarrow 0$. The irrational rotation $T x=\{x+\alpha\}$ admits cyclic approximation with speed $o(f(n))$ iff there exists a sequence of rational numbers $p / q \rightarrow \alpha$ such that

$$
|\alpha-p / q|=o(f(q)) .
$$

Proof. To prove the "if" part it clearly suffices to construct a sequence of Rokhlin towers $\tilde{\eta}_{n} \rightarrow \varepsilon$ of height $h_{n}$, such that the complement of the tower $\tilde{\eta}_{n}$ has measure $o\left(f\left(h_{n}\right)\right)$. We are going to select a subsequence $m_{n}$ and then construct $\tilde{\eta}_{n}$ out of the pair $\zeta_{m_{n}}, \zeta_{m_{n}}^{\prime}$ by forming a new base from levels of the two towers.

Without loss of generality we may assume that the $p / q$ are continued fraction convergents of $\alpha$ and $q f(q) \rightarrow 0$. Denote $M(q)=[1 / q f(q)]$. We start by choosing an arbitrary sequence $\varepsilon_{n}$ decreasing to zero. By compactness, we can find integers $K_{n}$ such that for every $x \in[0,1)$ there is a $j$ with $0 \leq j<K_{n}$ and $T^{j} x \in\left[0, \varepsilon_{n} / 2\right)$. Now choose $N_{n}$ such that $K_{n} / N_{n}<1 / n$. It is clear that for $k_{n}$ large enough we have

$$
N_{n} M\left(q_{k_{n}}\right)\left\|q_{k_{n}} \alpha\right\|<\varepsilon_{n} / 2
$$

and $K_{n}<q_{k_{n}}$. Finally choose $m_{n}$ satisfying

$$
M\left(q_{k_{n}}\right) q_{k_{n}}^{2} / q_{m_{n}}<1 / n .
$$

For the sake of convenience we may assume that the numbers $k_{n}, m_{n}$ are even and the sequences $k_{n}, m_{n}$ are increasing.

Now we form two new towers $\eta_{n}, \eta_{n}^{\prime}$ of height $q_{k_{n}}$. We construct the base $A$ of $\eta_{n}$ by selecting certain levels of $\zeta_{m_{n}}$. The first group of $N_{n} M\left(q_{k_{n}}\right)$ many levels are

$$
J_{0}, J_{q_{k_{n}}}, \ldots, J_{\left(N_{n} M\left(q_{k_{n}}\right)-1\right) q_{k_{n}}} .
$$

Each of them is clearly contained in $\left[0, \varepsilon_{n}\right)$. Now by the choice of $K_{n}$ we can find $s_{1}$ with $N_{n} M\left(q_{k_{n}}\right) q_{k_{n}} \leq s_{1}<N_{n} M\left(q_{k_{n}}\right) q_{k_{n}}+K_{n}$ such that $J_{s_{1}} \subset\left[0, \varepsilon_{n} / 2\right)$. The next group of selected levels are

$$
J_{s_{1}}, J_{s_{1}+q_{k_{n}}}, \ldots, J_{s_{1}+\left(N_{n} M\left(q_{k_{n}}\right)-1\right) q_{k_{n}}} .
$$


We continue up the tower in the same manner until we define the last group,

$$
J_{s_{p}}, J_{s_{p}+q_{k_{n}}}, \ldots, J_{s_{p}+(M-1) q_{k_{n}}},
$$

where $1 \leq M \leq N_{n} M\left(q_{k_{n}}\right)$, with at least $q_{k_{n}}-1$ but less than $2 q_{k_{n}}+K_{n}-2<$ $3 q_{k_{n}}-2$ top levels of $\zeta_{m_{n}}$ left over. The set

$$
\begin{aligned}
A= & J_{0} \cup J_{q_{k_{n}}} \cup \cdots \cup J_{\left(N_{n} M\left(q_{k_{n}}\right)-1\right) q_{k_{n}}} \\
& \cup \cdots \cup J_{s_{1}} \cup J_{s_{1}+q_{k_{n}}} \cup \cdots \cup J_{s_{1}+\left(N_{n} M\left(q_{k_{n}}\right)-1\right) q_{k_{n}}} \\
& \cup \cdots \cup J_{s_{p}} \cup J_{s_{p}+q_{k_{n}}} \cup \cdots \cup J_{s_{p}+(M-1) q_{k_{n}}}
\end{aligned}
$$

is the base of $\eta_{n}$. This new tower is contained entirely in $\zeta_{m_{n}}$ and only a small part of measure $\delta_{n}$ of the tower is not covered by $\eta_{n}$. Since less than $K_{n}$ levels have been skipped over every $N_{n} M\left(q_{k_{m}}\right) q_{k_{n}}$ th step and less than $2 q_{k_{n}}$ levels have been left on top, we obtain

$$
\delta_{n}<\frac{K_{n}}{N_{n} M\left(q_{k_{n}}\right) q_{k_{n}}}+\frac{2 q_{k_{n}}}{q_{m_{n}+1}} .
$$

Moreover, we have $A \subset\left[0, \varepsilon_{n}\right)$ by the choice of $k_{n}$.

We repeat the construction to form $\eta_{n}^{\prime}$. By selecting levels as before we obtain the base

$$
\begin{aligned}
A^{\prime}= & J_{0}^{\prime} \cup J_{q_{k_{n}}}^{\prime} \cup \cdots \cup J_{\left(N_{n} M\left(q_{k_{n}}\right)-1\right) q_{k_{n}}^{\prime}}^{\prime} \\
& \cup \cdots \cup J_{s_{1}^{\prime}}^{\prime} \cup J_{s_{1}^{\prime}+q_{k_{n}}}^{\prime} \cup \cdots \cup J_{s_{1}^{\prime}+\left(N_{n} M\left(q_{k_{n}}\right)-1\right) q_{k_{n}}}^{\prime} \\
& \cup \cdots \cup J_{s_{r}^{\prime}}^{\prime} \cup J_{s_{r}^{\prime}+q_{k_{n}}}^{\prime} \cup \cdots \cup J_{s_{r}^{\prime}+\left(M^{\prime}-1\right) q_{k_{n}} .}^{\prime}
\end{aligned}
$$

Clearly $A^{\prime} \subset\left(-\varepsilon_{n}, \varepsilon_{n}\right) ; \eta_{n}^{\prime}$ is contained in $\zeta_{m_{n}}^{\prime}$ and covers it up to

$$
\delta_{n}^{\prime}<\frac{K_{n}}{N_{n} M\left(q_{k_{n}}\right) q_{k_{n}}}+\frac{2 q_{k_{n}}}{q_{m_{n}}} .
$$

Finally we form a single tower $\tilde{\eta}_{n}$ by joining $\eta_{n}$ with $\eta_{n}^{\prime}$. Its base is $\tilde{A}=A \cup A^{\prime}$, and the height is $q_{k_{n}}$. It covers the space up to

$$
\tilde{\delta}_{n}=\delta_{n}+\delta_{n}^{\prime}<\frac{6}{n M\left(q_{k_{n}}\right) q_{k_{n}}}=o\left(f\left(q_{k_{n}}\right)\right) \text {. }
$$

Moreover, $\tilde{A} \subset\left(-\varepsilon_{n}, \varepsilon_{n}\right)$, so each level of $\tilde{\eta}_{n}$ has diameter at most $2 \varepsilon_{n}$ and consequently $\tilde{\eta}_{n} \rightarrow \varepsilon$, which ends the proof of sufficiency.

To prove the "only if" part assume there exist partitions

$$
\xi_{n}=\left\{C_{0}, \ldots, C_{h_{n}-1}\right\} \rightarrow \varepsilon
$$

and cyclic approximations $T_{n}$ of $T$ such that

$$
\sum_{j=0}^{h_{n}-1} \mu\left(T C_{j} \Delta T_{n} C_{j}\right)<\tilde{f}\left(h_{n}\right),
$$

where $\tilde{f}(n)=o(f(n))$. Let $\chi(x)=e^{2 \pi i x}$ on $[0,1)$. As $\xi_{n} \rightarrow \varepsilon$, we can find, for any $n$ large enough, an approximation $\chi_{n}=\sum_{j=0}^{h_{n}-1} \lambda_{j} 1_{C_{j}}$ of $\chi$, where $\left|\lambda_{j}\right|=1$ and $\left\|\chi_{n}-\chi\right\|<1 / 4$ in $L^{1}$. Observe that

$$
\left\|\chi_{n} \circ T_{n}^{-1}-\chi_{n} \circ T^{-1}\right\|=\left\|\sum \lambda_{j}\left(1_{T_{n} C_{j}}-1_{T C_{j}}\right)\right\| \leq \sum \mu\left(T_{n} C_{j} \Delta T C_{j}\right)<\tilde{f}\left(h_{n}\right) .
$$


For every $k \geq 1$ the same argument applies to the function $\chi_{n} \circ T_{n}^{-k}$ which is also of the form $\sum \lambda_{j}^{(k)} 1_{C_{j}}$, so we get by induction

$$
\begin{aligned}
\left\|\chi_{n} \circ T_{n}^{-k}-\chi_{n} \circ T^{-k}\right\| & \leq\left\|\left(\chi_{n} \circ T_{n}^{-k+1}\right) \circ T_{n}^{-1}-\left(\chi_{n} \circ T_{n}^{-k+1}\right) \circ T^{-1}\right\| \\
& +\left\|\chi_{n} \circ T_{n}^{-k+1}-\chi_{n} \circ T^{-k+1}\right\| \\
& <\tilde{f}\left(h_{n}\right)+(k-1) \tilde{f}\left(h_{n}\right)=k \tilde{f}\left(h_{n}\right) .
\end{aligned}
$$

Now by the triangle inequality we obtain

$$
\begin{aligned}
\left|e^{2 \pi i k \alpha}-1\right|= & \left\|\chi \circ T^{-k}-\chi\right\| \\
\leq & \left\|\chi \circ T^{-k}-\chi_{n} \circ T^{-k}\right\|+\left\|\chi_{n} \circ T^{-k}-\chi_{n} \circ T_{n}^{-k}\right\| \\
& +\left\|\chi_{n} \circ T_{n}^{-k}-\chi_{n}\right\|+\left\|\chi_{n}-\chi\right\| \\
& <k \tilde{f}\left(h_{n}\right)+\left\|\chi_{n} \circ T_{n}^{-k}-\chi_{n}\right\|+1 / 2 .
\end{aligned}
$$

In particular, for $k=l h_{n}, l=1,2, \ldots$, we have $\left|e^{2 \pi i l h_{n} \alpha}-1\right|<l h_{n} \tilde{f}\left(h_{n}\right)+1 / 2$, which implies $\left\|l h_{n} \alpha\right\|<l h_{n} \tilde{f}\left(h_{n}\right) / 4+1 / 8$. Notice that $\|l x\|=l\|x\|$ whenever $\|x\| \leq 1 / 2 l$. Consequently, by choosing $1 / 4\left\|h_{n} \alpha\right\| \leq l \leq 1 / 2\left\|h_{n}\right\|$ we get on dividing by $l$ that $\left\|h_{n} \alpha\right\|<h_{n} \tilde{f}\left(h_{n}\right) / 2$. Therefore, there exist integers $p_{n}$ such that $\left|\alpha-p_{n} / h_{n}\right|<\tilde{f}\left(h_{n}\right) / 2=o\left(f\left(h_{n}\right)\right)$, which ends the proof of the theorem.

It is well known that for every irrational number the rational approximation of Theorem 1 is satisfied with $f(n)=1 / n$ (and even $c_{n} / n^{2}$ for any $c_{n} \rightarrow \infty$ ), so every irrational rotation admits good cyclic approximation (see [2]). It is also clear that if $\alpha$ has unbounded partial quotients then we may take $f(n)=1 / n^{2}$. An even better approximation is possible for almost all numbers $\alpha$ (which also form a residual subset of the unit interval).

Let $g(x)>0$ for $x>0$ be such that the function $x g(x)$ is nonincreasing and $\int_{0}^{\infty} g(x) d x=\infty$. Then the inequality $|\alpha-p / q|<g(q) / q$ has infinitely many solutions for a.e. $\alpha$ (see [6, Theorem 32]). Consequently, the conditions of Theorem 1 are satisfied if $g(n) / n=o(f(n))$. In particular, if $g(x)=$ $(x \log x \log \log x)^{-1}$ for $x>3$ then $g(n) / n=o\left(1 / n^{2} \log n\right)$. We have obtained the following

Corollary. For a.e. $\alpha$ the rotation $T$ admits cyclic approximation with speed $o\left(1 / n^{2} \log n\right)$.

\section{Application}

Let $G$ denote a compact metrizable monothetic group with normalized Haar measure. If $T$ is an invertible measure-preserving transformation of $X$ and $\phi: X \rightarrow G$ a measurable function (called cocycle), we can define a skew product extension $T_{\phi}$ of $T$ acting on the product space by $T_{\phi}(x, g)=(T x, g+\phi(x))$. Denote by $\Phi$ the space of all cocycles endowed with topology of convergence in measure (cocycles that are equal a.e. are identified). It has been shown in [3] that if $T$ admits an approximation with speed $o(f(n))$ where $\sup f(n) / f(2 n)<\infty$ then, for generic $\phi$, the same is true of $T_{\phi}$. Here "generic" means from a residual subset of $\Phi$. In particular, $T_{\phi}$ is generically rank-1 if $f(n)=1 / n$ (similar results on simple spectrum have been obtained earlier by Robinson $[7,8])$. 
In the case where $X=G=T$, the circle group, and $T$ an irrational rotation of $\mathbf{T}, T_{\phi}$ is referred to as Anzai skew product; in multiplicative notation

$$
T_{\phi}(z, w)=\left(e^{2 \pi i \alpha} z, \phi(z) w\right) .
$$

The following result, which is now a consequence of Theorem 1 and Corollary, is an improved version of Corollary 2 in [3].

Theorem 2. For every irrational rotation generic Anzai skew products have good cyclic approximation. In particular, they are rank-1. Moreover, for a.e. rotation a generic Anzai skew product admits cyclic approximation with speed $o\left(1 / n^{2} \log n\right)$.

\section{REFERENCES}

1. A. del Junco, Transformations with discrete spectrum are stacking transformations, Canad. J. Math. 28 (1976), 836-839.

2. A. del Junco, A. Fieldsteel, and K. Park, Residual properties of $\alpha$-flows, preprint.

3. A. Iwanik and J. Serafin, Most monothetic extensions are rank-1, Colloq. Math. 66 (1993), 63-76.

4. A. Katok, Constructions in ergodic theory, preprint.

5. A. Katok and A. Stepin, Approximations in ergodic theory, Uspekhi Mat. Nauk 22 (1967), 81-106; English transl., Russian Math. Surveys 22 (1967), 77-102.

6. A. Ya. Khintchin, Continued fractions, Univ. of Chicago Press, Chicago, 1964.

7. E. A. Robinson, Ergodic measure preserving transformations with arbitrary finite spectral multiplicities, Invent. Math. 72 (1983), 299-314.

8. _ Non-Abelian extensions have nonsimple spectrum, Compositio Math. 65 (1988), 155-170.

Institute of Mathematics, Technical University of Wroclaw, 50-370 Wroclaw, Poland

E-mail address: iwanik@math.impwr.wroc.edu.pl 